\title{
Asymptotic Error Estimates for the Gauss Quadrature Formula
}

\author{
By M. M. Chawla and M. K. Jain
}

1. Introduction. Classical error estimates for the Gauss quadrature formula using derivatives can be used, but they are not of great practical value since the derivatives are not usually available. Davis and Rabinowitz [1] give a more convenient method for obtaining an upper bound for the error in the quadrature of analytic functions. McNamee [2] has discussed complex-variable methods for obtaining upper bounds for the errors of the Gaussian quadratures applied to analytic functions, and Barrett [3] has discussed their convergence.

The error of the $n$-point Gauss quadrature depends on $n$ and also on the function to be integrated. The object of the present paper is to obtain asymptotic estimates for the error of the Gauss quadrature formula, for large $n$, according to the nature of the integrand $f(x)$. The analysis also brings out the effect of the nature of $f(z)$ on the rate of convergence of the Gauss quadrature formula.

2. The Gauss Quadrature Formula. Let $C$ be a closed contour in the complex plane enclosing the interval $[-1,1]$ in its interior and let $f(z)$ be regular within $C$ and continuous in the closure of $C$. Denoting the zeros of the Legendre polynomial $P_{n}(x)$ defined on $[-1,1]$ by $\left\{x_{k}\right\}_{1}{ }^{n}$, on applying the residue theorem to the contour integral

$$
\frac{1}{2 \pi i} \int_{C} \frac{f(z) d z}{(z-x) P_{n}(z)}
$$

we get

$$
f(x)=\sum_{k=1}^{n} \frac{P_{n}(x)}{\left(x-x_{k}\right) P_{n}^{\prime}\left(x_{k}\right)} f\left(x_{k}\right)+\frac{1}{2 \pi i} \int_{C} \frac{f(z) P_{n}(x) d z}{(z-x) P_{n}(z)} .
$$

Integrating both sides with respect to $x$ over $[-1,1]$, we get

$$
\int_{-1}^{1} f(x) d x=\sum_{k=1}^{n} \lambda_{k} f\left(x_{k}\right)+E_{n}(f) \text {. }
$$

This is the Gaussian integration rule of order $n$ over the interval $[-1,1]$ with weights $\lambda_{k}=\left[P_{n}^{\prime}\left(x_{k}\right)\right]^{-1} \int_{-1}^{1} P_{n}(x) d x /\left(x-x_{k}\right)$. The abscissas $x_{k}$ and weights $\lambda_{k}$ have been tabulated extensively in [4], [5].

The error of the Gauss quadrature formula is given by

$$
E_{n}(f)=\frac{1}{\pi i} \int_{C} \frac{Q_{n}(z)}{P_{n}(z)} f(z) d z
$$

where $Q_{n}(z)=\frac{1}{2} \int_{-1}^{1} P_{n}(x) d x /(z-x)$ is the Legendre function of the second kind. See also Davis [6, p. 361].

Received October 19, 1966. Revised May 22, 1967. 
3. An Asymptotic Formula for $E_{n}(f)$. We now put the formula for the error in an asymptotic form for large $n$. An asymptotic expression for $Q_{n}(z) / P_{n}(z)$, for large $n$, is given (Barrett [3]) by

$$
\frac{Q_{n}(z)}{P_{n}(z)} \simeq \frac{\pi}{\left(z \pm\left(z^{2}-1\right)^{1 / 2}\right)^{2 n+1}},
$$

where the sign is chosen so that $\left|z \pm\left(z^{2}-1\right)^{1 / 2}\right|>1$, and (5) is valid in the $z$ plane with the interval $[-1,1]$ removed. Observe that $Q_{n}(-z) / P_{n}(-z)=$ $-Q_{n}(z) / P_{n}(z)$.

Substituting (5) in (4) we obtain an asymptotic formula for the error

$$
E_{n}(f) \simeq-i \int_{C} \frac{f(z) d z}{\left(z+\left(z^{2}-1\right)^{1 / 2}\right)^{2 n+1}}
$$

valid for large $n$. In the following we shall be concerned with the estimation of the contour integral in (6) according to the behaviour of $f(z)$.

A similar integral has been estimated, for large $n$, depending on the nature of $f(z)$, in connection with the asymptotic estimation of the coefficients in the Chebyshev series expansion of a function, by Elliott [7] and by Elliott and Szekeres [8].

3.1. Entire Functions. In this section we consider the estimation of the error $E_{n}(f)$ of the Gauss quadrature applied to entire functions.

Observe that for entire $f(z)$, the contour in (6) can be displaced freely in the plane, provided only that it never crosses the branch points $z= \pm 1$. We can therefore use the method of steepest descents to estimate $E_{n}(f)$, when the contour is deformed to pass through the saddle points of the integrand.

As in [8], we write the integrand in (6) as $\exp (\psi(z))$ where

$$
\psi(z)=\log f(z)-(2 n+1) \log \left(z+\left(z^{2}-1\right)^{1 / 2}\right) .
$$

Assuming that the main contribution to the contour integral, for large $n$, comes from the portion of the integral passing through a saddle point (where $\psi^{\prime}(\zeta)=0$ ), we obtain the estimate of error,

$$
E_{n}(f) \simeq-i(2 \pi)^{1 / 2} \alpha\left|\psi^{\prime \prime}(\zeta)\right|^{-1 / 2} \exp (\psi(\zeta))
$$

where $|\alpha|=1$ and $\arg \alpha=\pi / 2-\frac{1}{2} \arg \psi^{\prime \prime}(\zeta)$.

For example, for the function $\exp (x),(7)$ gives the error estimate,

$$
E_{n}(f) \simeq \frac{(2 \pi)^{1 / 2}(2 n+1) \exp (\zeta)}{\zeta^{1 / 2}\left(\zeta+\left(\zeta^{2}-1\right)^{1 / 2}\right)^{2 n+1}},
$$

where $\zeta=\left(1+(2 n+1)^{2}\right)^{1 / 2}$.

\section{TABLE $1^{*}$}

\begin{tabular}{ccc}
\hline$n$ & Estimated $E_{n}$ & Actual $E_{n}$ \\
\hline 5 & $8.698(-10)$ & $8.248(-10)$ \\
6 & $1.641(-12)$ & $1.568(-12)$ \\
\hline
\end{tabular}

* Values in the parentheses indicate the power of 10 by which the tabulated values should be multiplied. 
TABLE 1a

\begin{tabular}{llll}
\hline$n$ & Estimated $E_{n}$ & Actual $E_{n}$ & $\begin{array}{c}\text { Upper bound } \\
\text { for }\left|E_{n}\right|\end{array}$ \\
\hline 5 & $6.387(-7)$ & $6.041(-7)$ & $4.0(-6)$ \\
6 & $2.195(-9)$ & $2.094(-9)$ & $2.0(-8)$ \\
7 & $4.955(-12)$ & $4.758(-12)$ & $4.0(-11)$ \\
\hline
\end{tabular}

A comparison of the estimated with the actual error for exp $(x)$ is given in Table 1. As a second example, consider $f(x)=x^{3} \exp (x)$. Table 1a gives a comparison of the actual error for this function with that estimated from (7), and with the upper bounds for $E_{n}$ obtained by McNamee [2] (last column).

3.2. Function with Pole. Let $\varepsilon_{\rho}(\rho>1)$ designate the ellipse whose foci are at $z= \pm 1$ and whose semi-axes are respectively $\frac{1}{2}\left(\rho+\rho^{-1}\right)$ and $\frac{1}{2}\left(\rho-\rho^{-1}\right)$.

Suppose that $f(z)$ has a pole of order $k$ at $z=z_{0}$, say $f(z)=A_{k} /\left(z-z_{0}\right)^{k}$ where $A_{k}$ is constant. In (6), let the contour be selected to be an ellipse $\varepsilon_{\rho}$ described in the positive sense and enclosing $z_{0}$ in its interior, joined by a cross-cut (not intersecting the interval $[-1,1])$ to a small circle with centre at $z_{0}$, described in the negative sense. Now, let $\rho \rightarrow \infty$ and let the radius of the small circle tend to zero. Assuming that $f(z)$ is such that the integral around $\varepsilon_{\rho}$ tends to zero as $\rho \rightarrow \infty$, for large $n$, from (6) we obtain

$$
E_{n}(f) \simeq-2 \pi \frac{A_{k}}{(k-1) !} \frac{d^{k-1}}{d z^{k-1}}\left[\frac{1}{\left(z+\left(z^{2}-1\right)^{1 / 2}\right)^{2 n+1}}\right]_{z=z_{0}} .
$$

An estimate of error for $f(z)$ having a simple pole at $z=z_{0}$ follows immediately from (9). In case $f(z)$ has simple poles away from the real axis, say at $z_{0}$ and $\bar{z}_{0}$ with residues $A_{0}$ and $\bar{A}_{0}$, we easily obtain the estimate

$$
E_{n}(f) \simeq-4 \pi \operatorname{Re}\left[\frac{A_{0}}{\left(z_{0}+\left(z_{0}^{2}-1\right)^{1 / 2}\right)^{2 n+1}}\right] .
$$

See also Barrett [3, p. 273]. As an example, consider the function $f(x)=\left(9 x^{2}+1\right)^{-1}$ for which (10) gives the error estimate

$$
E_{n} \simeq(-1)^{n} 2 \pi\left[\frac{3}{1+(10)^{1 / 2}}\right]^{2 n+1} .
$$

TABLE 2

\begin{tabular}{rrr}
\hline$n$ & Estimated $E_{n}$ & \multicolumn{1}{c}{ Actual $E_{n}$} \\
\hline 5 & $-5.714(-2)$ & $-5.787(-2)$ \\
6 & $2.968(-2)$ & $2.891(-2)$ \\
7 & $-1.542(-2)$ & $-1.537(-2)$ \\
8 & $8.011(-3)$ & $7.904(-3)$ \\
9 & $-4.161(-3)$ & $-4.134(-3)$ \\
10 & $2.162(-3)$ & $2.143(-3)$ \\
11 & $-1.123(-3)$ & $-1.116(-3)$ \\
12 & $5.834(-4)$ & $5.794(-4)$ \\
16 & $4.249(-5)$ & $4.227(-5)$ \\
\hline
\end{tabular}


The estimated error is compared in Table 2 with the actual error.

To estimate (9) for $k \geqq 2$, on performing one differentiation, it can be put in the form

$$
E_{n}(f) \simeq 2 \pi(2 n+1)\left(A_{k} /(k-1) !\right) g^{(k-2)}\left(z_{0}\right),
$$

where we have put

$$
g(z)=\frac{1}{\left(z^{2}-1\right)^{1 / 2}\left(z+\left(z^{2}-1\right)^{1 / 2}\right)^{2 n+1}} .
$$

Substituting for $g^{(k-2)}(z)$ from [7, p. 278], we find when $\operatorname{Re} z_{0}>1$ and subject to the condition that $\left|z_{0} /\left(z_{0}^{2}-1\right)^{1 / 2}-1\right|<2$

$$
E_{n}(f) \simeq \frac{2 \pi}{(2 n) !} \frac{A_{k}}{(k-1) !} \frac{(-1)^{k}(2 n+k-1) !}{\left(z_{0}^{2}-1\right)^{(k-1) / 2}\left(z_{0}+\left(z_{0}{ }^{2}-1\right)^{1 / 2}\right)^{2 n+1}},
$$

and in case $\operatorname{Re} z_{0}<-1$, the estimate (14) is to be multiplied by -1 .

For $\left|\operatorname{Re} z_{0}\right|<1$, again substituting for $g^{(k-2)}\left(z_{0}\right)$ from [7, p. 279], we obtain

$$
\begin{aligned}
E_{n}(f) \simeq & 2 \pi(2 n+1) \frac{A_{k}}{(k-1) !} \frac{(-1)^{k} e^{\mp(n+1 / 2) i \pi}(2 n+k-1) !}{\left(z_{0}{ }^{2}-1\right)^{(k-1) / 2}} \\
& \times P_{k-2}^{-(2 n+1)}\left(\frac{z_{0}}{\left(z_{0}{ }^{2}-1\right)^{1 / 2}}\right),
\end{aligned}
$$

where the upper or the lower sign is taken according as $\operatorname{Im} z_{0} \gtrless 0$, and $P_{k-2}^{-(2 n+1)}$ is the Legendre function of the first kind.

3.3. Function with Singularity on the Real Axis. First, we consider the case of a function having a branch point on the real axis.

Assume that $f(z)=(c-z)^{\phi} g(z),(c>1)$ where $\phi$ is nonintegral and $g(z)$ is regular at $z=c$. To estimate the contour integral in (6), we choose the contour as in Section 3.2 except that the cross-cut now encloses the part of the real axis between the small circle, centered at $z=c$, and the ellipse. We assume again that the integral in (6) along $\varepsilon_{\rho}$ tends to zero as $\rho \rightarrow \infty$; and $\phi>-1$ so that the integral around the small circle tends to zero. In the limit, therefore, the only contributions to the contour integral in (6) come from the line segments of the crosscut, which combine to give

$$
E_{n}(f) \simeq-2 \sin (\pi \phi) \lim _{a \rightarrow \infty} \int_{c}^{a} \frac{|c-x|^{\phi} g(x) d x}{\left(x+\left(x^{2}-1\right)^{1 / 2}\right)^{2 n+1}} .
$$

The integral in (16) can be estimated as in [7, p. 282]. Assuming that $g(z)$ is such that, for large $n$, the main contribution to the integral comes from values close to $z=c$, we obtain the estimate

$$
E_{n}(f) \simeq-2 \sin (\pi \phi)\left(c^{2}-1\right)^{(\phi+1) / 2} \frac{g(c) \Gamma(\phi+1)}{(2 n+1)^{\phi+1}\left(c+\left(c^{2}-1\right)^{1 / 2}\right)^{2 n+1}} .
$$

In case $f(z)$ has a branch point at $z=-c(c>1)$, the estimate (20) holds with the sign changed.

The above analysis can be used to obtain estimates of error for a function 
regular everywhere except at the end-points of the interval of integration. Let $f(z)=(1-z)^{\phi} g(z)$. Since $f(z)$ has to be bounded at $z=1$, we must have $\phi>0$. Under similar assumptions as above, we obtain in this case the estimate

$$
E_{n}(f) \simeq-\frac{2^{1-\phi} \sin (\pi \phi) g(1)}{(2 n+1)^{2(\phi+1)}} \Gamma(2 \phi+2) .
$$

For $f(z)$ with only singularity of the above type at $z=-1$, the estimate (21) holds with the sign changed.

As an illustration, consider the function $(1+x)^{1 / 2}$, for which (21) gives the error estimate

$$
E_{n} \simeq \frac{2 \sqrt{ } 2}{(2 n+1)^{3}} .
$$

The estimated and the actual errors in the Gauss quadrature formula for the function $(1+x)^{1 / 2}$ are compared in Table 3 .

\section{TABLE 3}

\begin{tabular}{rcc}
\hline$n$ & Estimated $E_{n}$ & Actual $E_{n}$ \\
\hline 5 & $2.125(-3)$ & $1.782(-3)$ \\
6 & $1.287(-3)$ & $1.074(-3)$ \\
7 & $8.381(-4)$ & $6.970(-4)$ \\
8 & $5.757(-4)$ & $4.778(-4)$ \\
9 & $4.066(-4)$ & $3.417(-4)$ \\
10 & $3.054(-4)$ & $2.528(-4)$ \\
11 & $2.325(-4)$ & $1.923(-4)$ \\
12 & $1.810(-4)$ & $1.496(-4)$ \\
16 & $6.906(-5)$ & $6.495(-5)$ \\
\hline
\end{tabular}

Next, error estimates for a function having a logarithmic singularity on the real axis can also be obtained by the above method. Let $f(z)=g(z) \log (c-z)(c>1)$, and let $f(z)$ and $g(z)$ satisfy the same conditions as above. Since the integral around the small circle tends to zero as the radius of the circle tends to zero, the contributions from the cross-cut in this case combine to give

$$
E_{n}(f) \simeq-2 \pi \lim _{a \rightarrow \infty} \int_{c}^{a} \frac{g(x) d x}{\left(x+\left(x^{2}-1\right)^{1 / 2}\right)^{2 n+1}} .
$$

Calculating the integral as above, we obtain the estimate

$$
E_{n}(f) \simeq-\frac{2 \pi g(c)\left(c^{2}-1\right)^{1 / 2}}{(2 n+1)\left(c+\left(c^{2}-1\right)^{1 / 2}\right)^{2 n+1}} .
$$

In case $c$ lies on the negative real axis $(c<-1)$, the estimate (21) holds with the sign changed. As an example, consider $f(x)=\log (1.1+x)$ for which (21) gives the error estimate

$$
E_{n} \simeq \frac{2 \pi(0.21)^{1 / 2}}{(2 n+1)\left(1.1+(0.21)^{1 / 2}\right)^{2 n+1}}
$$


Table 4 gives a comparison of the estimated with the actual error for this function.

TABLE 4

\begin{tabular}{rll}
\hline$n$ & Estimated $E_{n}$ & Actual $E_{n}$ \\
\hline 5 & $1.991(-3)$ & $2.212(-3)$ \\
6 & $6.938(-4)$ & $7.591(-4)$ \\
7 & $2.476(-4)$ & $2.678(-4)$ \\
8 & $8.998(-5)$ & $9.647(-5)$ \\
9 & $3.316(-5)$ & $3.529(-5)$ \\
10 & $1.235(-5)$ & $1.307(-5)$ \\
11 & $4.646(-6)$ & $4.893(-6)$ \\
12 & $1.760(-6)$ & $1.846(-6)$ \\
16 & $3.836(-8)$ & $3.977(-8)$ \\
\hline
\end{tabular}

4. Extension to the Gauss-Jacobi Quadratures. Finally, we indicate that the above methods can be used to obtain asymptotic error estimates for the GaussJacobi quadratures. The Gauss-Jacobi quadrature formula

$$
\int_{-1}^{1}(1-x)^{\alpha}(1+x)^{\beta} f(x) d x=\sum_{k=1}^{n} \lambda_{k} f\left(x_{k}\right)+E_{n}(f)
$$

has been described in Barrett [3], and an asymptotic formula for the error is given in the form

$$
E_{n}(f) \simeq-i \int_{C}(z-1)^{\alpha}(z+1)^{\beta} \frac{f(z) d z}{\left(z+\left(z^{2}-1\right)^{1 / 2}\right)^{(2 n+\alpha+\beta+1)}} .
$$

Estimates for $E_{n}(f)$ can now be obtained from (24).

5. Conclusion. In this paper we have obtained estimates for Gaussian quadrature errors for large $n$. The form of the estimate depends upon the nature of the integrand $f(z)$. The analysis also brings out the effect of the nature of $f(z)$ on the rate of convergence of the Gaussian quadrature. For instance, Eq. (19) indicates that Gaussian integration can be relatively slow in convergence. Even entire functions can behave quite wildly and the behaviour of the function in the neighbourhood of saddle points may be quite inadequate as an estimate of the function over a domain of the complex plane.

Acknowledgements. We wish to thank the referees for their helpful comments and suggestions. We also wish to thank Professor Philip J. Davis for communicating this paper.

Department of Mathematics Indian Institute of Technology Hauz Khas, New Delhi-29 India

1. P. J. Davis \& P. Rabinowitz, "On the estimation of quadrature errors for analytic functions," $M T A C$, v. 8, 1954, pp. 193-203. MR 16, 404. 
2. J. McN formula and by Gauss-type formulae," Math. Comp., v. 18, 1964, pp. 368-381. MR 32 \#3264.

3. W. BARRETT, "Convergence properties of Gaussian quadrature formulae," Comput. J., v. $3,1960 / 61$, pp. 272-277. MR 23 \#B1117.

4. P. Davis \& P. Rabinowitz, "Abscissas and weights for Gaussian quadratures of high order," J. Res. Nat. Bur. Standards, v. 56, 1956, pp. 35-37. MR 17, 902.

5. P. Davis \& P. RABINOwITZ, "Additional abscissas and weights for Gaussian quadratures of high order,"J. Res. Nat. Bur. Standards, v. 60, 1956, pp. 613-614.

6. P. J. DAvis, Interpolation and Approximation, Blaisdell, New York, 1963. MR 28 \#393.

7. D. ELLIOTT, "The evaluation and estimation of the coefficients in the Chebyshev series expansion of a function," Math. Comp., v. 18, 1964, pp. 274-284. MR 29 \#4176.

8. D. ElliotT \& G. SzEkEREs, "Some estimates of the coefficients in the Chebyshev series expansion of a function," Math. Comp., v. 19, 1965, pp. 25-32. MR 30 \#2666. 